(C) [2009] IEEE. Reprinted, with permission, from [Leijia Wu, Kumbesan Sandrasegaran, and Maged Elkashlan, A system level simulation model for common radio resource management, Communications, 2009. APCC 2009. 15th Asia-Pacific Conference on 8-10 Oct. 2009]. This material is posted here with permission of the IEEE. Such ermission of the IEEE does not in any way imply IEEE endorsement of any of the University of Technology, Sydney's products or services. Internal or personal use of this material is permitted. However, permission to reprint/republish this material for advertising or promotional purposes or for creating new collective works for resale or redistribution must be obtained from the IEEE by writing to pubs-permissions@ieee.org. By choosing to view this document, you agree to all provisions of the copyright laws protecting it 


\title{
A System Level Simulation Model for Common Radio Resource Management
}

\author{
Leijia Wu, Kumbesan Sandrasegaran, and Maged Elkashlan, Member, IEEE, \\ \{lewu,kumbes\}@eng.uts.edu.au, maged.elkashlan@csiro.au, \\ University of Technology, Sydney and Wireless Technologies Laboratory, CSIRO ICT Centre,
} Australia

\begin{abstract}
A major challenge of the future heterogeneous wireless network is the Radio Resource Management (RRM) strategy. The concept of Common RRM (CRRM) has been proposed to jointly manage radio resources among different Radio Access Technologies (RATs) in an optimized way. In order to evaluate the performance of different CRRM algorithms, a simulation model needs to be developed. This paper proposes a system level CRRM simulation model for integrated GSM/UMTS networks. In the proposed model, cell topology, user mobility, radio propagation loss, user traffic generation, and admission control are considered. The principles of system modeling and simulation presented in this paper can be used in future research on CRRM.
\end{abstract}

Index Terms-common radio resource management, radio access technology selection, system level modeling, performance evaluation

\section{INTRODUCTION}

$\mathrm{T}$ The next generation wireless network is expected to be a heterogeneous system, which integrates different Radio Access Technologies (RATs), e.g. GSM/EDGE Radio Access Network (GERAN), UMTS Terrestrial Radio Access Network (UTRAN) and Wireless Local Area Network (WLAN) through a common platform. A challenge arising from this heterogeneous network is the Radio Resource Management (RRM) strategy. Currently, Radio Resource Management (RRM) strategies are implemented in different kinds of networks separately. Each of them works well in the network that it is designed for. However, they are not suitable for the heterogeneous network. In order to solve this problem, Common RRM (CRRM) strategy has been proposed in the literature to coordinate radio resources among different RATs in an optimized way [1]. With a proper CRRM algorithm, a user can be allocated to the most suitable RAT. A number of CRRM algorithms have been studied in the literature [2].

Software simulation is an important tool for performance evaluation of CRRM algorithms. This is because manually analytical methods are not feasible for today's complicated telecommunication systems. For most researchers, it is also impractical to implement their tests on the real system. Telecommunication system simulation can be classified into two types: link level simulation and system level simulation. Link level simulation only focuses on the chip level or symbol level, where user locations may not be relevant. However, in system level simulation, user mobility and traffic characteristics have to be considered. Radio propagation and admission control are necessary to be included too.

This paper proposes a system level simulation model for CRRM in a combined GSM/UMTS network using MATLAB. The performance of different CRRM algorithms can be evaluated using this model. The rest of the paper is organized as follows. In Section II to VI, different function modules in the proposed model, such as cell topology, user mobility, radio propagation loss, user traffic models, and admission control algorithm are described respectively. The calculations of key performance evaluation parameters are presented in Section VII. Section VIII summarizes the overall CRRM simulation model that combines all the function modules and Section IX concludes this paper.

\section{CELL TOPOLOGY MODELING}

Cell topology modeling is used to determine radio coverage area and the location of Node B/Base Station (BS). The Node B or BS can be located at the central of every cell or at the common edges of adjacent cells. In our cell topology model, the former one is used. The traditional hexagonal cell topology is used.

The complete cell topology is shown in Fig. 1, where the dotted lines indicate the simulation area and the solid lines indicate user movement. There are seventeen hexagonal cells shown in Fig. 1. However, by using the wraparound technique, only twelve cells are in the simulation area because some half cells are considered as the same cell, such as cells 9 and 13. The details of the wraparound technique will be discussed in Section III. It is assumed that UMTS and GSM networks have exactly the same topology - one network exactly overlaps on another.

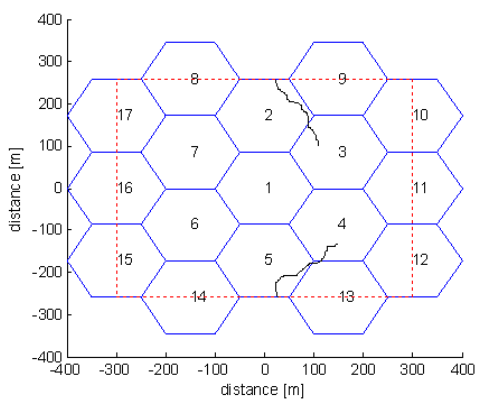

Fig. 1. Cell topology 


\section{USER MOBILITY MODELING}

In order to execute the simulation in a realistic scenario, it is assumed that the users are moving rather than static. The mobility model is used to update user positions based on certain user movement pattern. The input parameters of this model are user direction and user velocity. The mobility model is expressed in (1).

$$
\operatorname{position~}_{u}(t+1)=\operatorname{position}_{u}(t)+v_{u}(t) \cdot e^{i \cdot \theta_{u}(t)},
$$

where $v(t)$ and $\theta(t)$ are the user velocity and direction at time $t$ respectively. Index $u$ denotes the $u^{\text {th }}$ user. $\theta(t)$ is given by (2).

$$
\theta u(t)=\theta u(t-1)+\operatorname{rand}^{*} 2 * \pi, \quad \text { (2) }
$$

where rand is a random generated number between 0 and 1 .

In our simulation model, users are only allowed to move within a predefined simulation boundary. The border effect is alleviated by using the wraparound technique. The left and right borders and top and bottom borders are connected to each other. When a user reaches the simulation boundary, the user will be wrapped around to the opposite side.

\section{RADIO PROPAGATION LOSS MODELING}

In radio transmission, the signal strength decreases with the increase of the distance between the transmitter (Tx) and the receiver $(\mathrm{Rx})$. Different propagation models have been studied in the literature [3]. In our simulation, the well known Okumura-Hata propagation model has been selected, which is shown in (3):

$$
L_{d B}=A+B \cdot \log _{10}(d),
$$

where $A$ and $B$ are constants computed from a given set of parameters including BS antenna height, mobile station (MS) antenna height, and carrier frequency; $d$ is the distance between BS and MS.

If we assume that an urban macro cell with BS antenna height of $3 \mathrm{~m}$, MS antenna height of $1.5 \mathrm{~m}$ and carrier frequency of $1950 \mathrm{MHz}$, the Okumura-Hata propagation model can be written as [4]

$$
L_{d B}=137.4+35.2 \cdot \log _{10}(d),
$$

If we consider an additional log-normal shadowing with standard deviation of $10 \mathrm{~dB}$ for a urban macro-cell model [5], (4) becomes:

$$
L_{d B}=137.4+35.2 \cdot \log _{10}(d)+10 d B .
$$

\section{USER TRAFFIC MODELING}

In the proposed simulation model, the number of calls made by every user is generated according to a Poission arrival process, which is expressed in (6).

$$
f(k, \lambda)=\frac{\lambda^{k} e^{-\lambda}}{k !},
$$

where $k=0,1,2 \ldots$, is the number of arrival calls, $\lambda$ is the number of arrival calls during an given interval.

In MATLAB, there is a built in function to generate random Poission distribution numbers with a mean of $\lambda$, which is poissrnd $(\lambda)$.

The holding time of user calls is generated according to an exponentially distribution, which is expressed in (7).

$$
f(x ; \lambda)=\left\{\begin{array}{c}
\lambda e^{-\lambda x}, x \geq 0 \\
0, x<0 .
\end{array}\right.
$$

where $f(x ; \lambda)$ is the probability density function and $\lambda$ is the mean call duration.

In MATLAB, there is a built in function to generate randomly exponential distribution numbers with a mean of $\lambda$, which is exprnd $(\lambda)$.

\section{ADMISSION CONTROL ALGORITHM}

Admission control algorithms for GSM and UMTS are necessary in our simulation model to calculate the call blocking and dropping probabilities. The admission control for GSM is relatively simple. For GSM, it is assumed that there are three carrier frequencies in every cell. Each of them can be divided into eight time slots. So, a GSM cell has 24 physical channels in total. It is assumed that time slot 0 is reserved for the broadcast channel so that there are 21 channels available for user data transmission. A service request is rejected if all the GSM channels are occupied. Voice calls have higher priority over data calls.

For UMTS, a single FDD carrier frequency of $1950 \mathrm{MHz}$ with a bandwidth of $5 \mathrm{MHz}$ is considered. In UMTS, call acceptance depends on three factors: channelization code, load factor and BS transmission power. These factors will be discussed in this section.

In UMTS networks, channelization codes are generated from a code tree to maintain orthogonality so that the interference between different codes can be minimized. These codes are used for channel separation from a single source. The number of channelization codes is limited. A call will not be accepted if there is no free code to be allocated.

Load factor is a parameter introduced to measure system load. When a UMTS network is fully loaded, its load factor is 1. Because a UMTS system will be unstable if it is fully loaded (the powers of all users will reach to the maximum level in this case), a safety margin is required. Here the maximum allowed load factor value (load factor threshold) is set to be 0.75 . In UMTS networks, the uplink and downlink load factors should be calculated separately. The uplink load factor can be calculated by (8)[6]:

$$
\eta_{U L}=(1+f) \cdot \sum_{j=1}^{N} \frac{1}{1+\frac{W}{\left(E_{b} / N_{0}\right)_{j} \cdot R_{j} \cdot v_{j}}},
$$

where $\eta_{U L}$ is the uplink load factor, $f$ is the other cells to own cell interference ratio, $N$ is the number of service connections, $W$ is the WCDMA chip rate, $E_{b} / N_{0}$ is the signal energy per bit to noise spectral density ratio, $R_{j}$ is the bit rate and $v_{j}$ is the activity factor of a service at physical layer. The downlink load factor is given by (9) [6]:

$$
\eta_{D l}=\sum_{j=1}^{N} v_{j} \cdot \frac{\left(E_{b} / N_{0}\right)_{j}}{W / R_{j}} \cdot[(1-\bar{\alpha})+\bar{f}],
$$

where $\eta_{D L}$ is the downlink load factor, $\bar{a}$ is the average orthogonality factor in the cell and $\bar{f}$ is the average other cells to own cell interference ratio.

A new service request is accepted if in uplink 


$$
\text { New_ } \eta_{U L}<\eta_{U L_{-} \text {threshold }} \text {, }
$$

and the same in downlink:

$$
\text { New_ } \eta_{D L}<\eta_{D L_{-} \text {threshold }} \text {, }
$$

where $N e w \_\eta_{U L}$ and $N e w \_\eta_{D L}$ are the uplink and downlink load factors after accepting the new service request; $\eta_{U L \text {-threshold }}$ and $\eta_{D L-t h r e s h o l d}$ are the uplink and downlink load factor thresholds, respectively.

In the UMTS network, the BS transmission power is limited and part of the power is reserved for common channels. The BS transmission power calculation is given in (12) [7]:

$$
P_{D L}=\frac{P_{N} \cdot \sum_{j=1}^{n} \frac{\left(E_{b} / N_{0}\right)_{j} \cdot R_{j} \cdot v_{j}}{W} \cdot L_{j}}{1-\eta_{D L}},
$$

where $P_{D L}$ is the downlink BS transmission power for traffic channels, $P_{N}$ is the thermal noise power and $L$ is the loss between BS and User Equipment (UE) (including propagation loss and penetration loss). The propagation loss calculation has already described in (4). In order to simplify the problem, the penetration loss can be assumed to a constant, such as $20 \mathrm{~dB}$.

If a user wants to be accepted, in addition to meet the channelization code and load factor requirements, it also should meet the following power requirement

$$
\text { New_ } P_{D L}<P_{D L_{-} \max },
$$

where New $\_P_{D L}$ is the downlink transmission power after accepting the new user and $P_{D L_{-} \max }$ is the maximum BS transmission power allocated to traffic channels.

A service request is admitted only when it meets all the requirements described in (10), (11) and (13). Otherwise, the service request is blocked. The details of QoS requirements for different types of users and UMTS network parameters are shown in Table I and II, respectively.

TABLE I

QOS REQUIRMENTS FOR DIFFERNER TYPES OF USERS

\begin{tabular}{|l|l|l|l|}
\hline Parameters & Voice call (CBR) & $\begin{array}{l}\text { Video call } \\
\text { (CBR) }\end{array}$ & Data call (VBR) \\
\hline Activity factor $\mathrm{v}_{\mathrm{j}}$ & $\begin{array}{l}\text { Uplink: } 0.67 \\
\text { Downlink: } 0.58\end{array}$ & 1 & 1 \\
\hline $\mathrm{E}_{\mathrm{b}} / \mathrm{N}_{0}$ & $7 \mathrm{~dB}$ & $5 \mathrm{~dB}$ \\
\hline $\begin{array}{l}\text { Block error rate } \\
\text { BLER }\end{array}$ & $1 \%$ & $10 \%$ \\
\hline Bit rate of user $\mathrm{R}_{\mathrm{j}}$ & $\begin{array}{l}12.2 \text { kbps in both } \\
\text { directions }\end{array}$ & $\begin{array}{l}64 \text { kbps in } \\
\text { both } \\
\text { directions }\end{array}$ & $\begin{array}{l}\text { Uplink: up to } \\
64 \mathrm{kbps} \\
\text { Downlink: up to } \\
128 \mathrm{kbps}\end{array}$ \\
\hline
\end{tabular}

TABLE II

UMTS NETWORK PARAMETERS

\begin{tabular}{|l|l|}
\hline Uplink load factor threshold & 0.75 \\
\hline Downlink load factor threshold & 0.75 \\
\hline WCDMA chip rate $\mathrm{W}$ & $3.84 \mathrm{Mcps}$ \\
\hline Average orthogonality $\bar{\alpha}$ & 0.5 \\
\hline Other cells to own cell interference ratio $f$ & 0.65 \\
\hline Maximum base station transmission power & $20 \mathrm{~W}$ \\
\hline Common channel power allocation & $3 \mathrm{~W}$ \\
\hline Base station antenna height & $30 \mathrm{~m}$ \\
\hline Mobile antenna height & $1.5 \mathrm{~m}$ \\
\hline Carrier frequency & $1950 \mathrm{MHz}$ \\
\hline Thermal noise power & $-108 \mathrm{dbm}$ \\
\hline
\end{tabular}

\section{KEY PERFORMANCE PARAMETERS}

Call blocking/dropping probabilities and throughput are two of the most important parameters used to evaluate the performance of a CRRM algorithm. For Constant Bit Rate (CBR) services, such as voice calls and video calls, we are interested in their blocking and dropping probabilities, which can be easily found by counting the numbers of calls blocked and dropped and then dividing them by the total number of calls generated. For Variable Bit Rate (VBR) services, such as web browsing, we are interested in the user throughput. It should be noticed that CBR services always have higher priority over VBR services. VBR services share the resources not used by CBR services.

The user throughput calculation in GSM is simple. A data call can obtain a bit rate up to $14.4 \mathrm{kbps}$ in both directions [8]. The total bit rate of data users in GSM then equals to the number of data users served in GSM multiplying by 14.4 kbps.

In UMTS, the calculation is more complicated and we need to consider the uplink and downlink directions separately. The uplink bit rate for VBR services is calculated as follows. According to (8),

$$
R_{U L_{-} V B R}=\frac{\eta_{U L_{L} V B R} \cdot(1+W)}{v_{V B R} \cdot\left(E_{b} / N_{0}\right)_{V B R} \cdot(1+f)},
$$

where $R_{U L-V B R}$ is the total uplink bit rate for VBR services, $\eta_{U L-V B R}$ is the downlink load factor of VBR services, which can be calculated by

$$
\eta_{U L_{-} V B R}=\eta_{U L_{-} \text {threshold }}-\eta_{U L_{-} C B R} \text {, }
$$

where $\eta_{U L-C B R}$ is the uplink load factor of CBR services.

The calculation of downlink bit rate is more complex because both of the load factor and BS transmission power aspects have to be considered. Similar to the uplink one, according to (9),

$$
R_{D L_{-} V B R}=\frac{\eta_{D L_{-V B R}} \cdot W}{v_{V B R} \cdot\left(E_{b} / N_{0}\right)_{V B R} \cdot[(1-\bar{\alpha})+\bar{f}]},
$$

where $R_{D L-V B R}$ is the total downlink bit rate for VBR services, $\eta_{D L-V B R}$ is the downlink load factor of VBR services, which can be calculated from

$$
\eta_{D L_{-} V B R}=\eta_{D L_{-} \text {threshold }}-\eta_{D_{L_{-} C B R}} \text {, }
$$

where $\eta_{D L-C B R}$ is the downlink load factor of CBR services.

According to (12), the total downlink bit rate of VBR services is

$$
R_{D L_{-} V B R}=\frac{P_{D L_{V} V B R} \cdot W \cdot\left(1-\eta_{D L}\right)}{P_{N} \cdot\left(E_{b} / N_{0}\right)_{V B R} \cdot v_{V B R} \cdot \bar{L}},
$$

where $\overline{\boldsymbol{L}}$ is the average path loss of all served users and $P_{D L-V B R}$ is the power left for VBR services, which can be calculated as

$$
P_{D L_{-} V B R}=P_{D L_{-} \max }-P_{D L_{-} C B R},
$$

where $P_{D L-C B R}$ is the power allocated to CBR services.

The downlink bit rate of VBR users in UMTS is the minimum one calculated from (16) and (18). After considering the Block Error Rate (BLER), the UMTS downlink VBR service throughput is given by

$$
\text { Throughput }=R \cdot(1-B L E R) \text {. }
$$




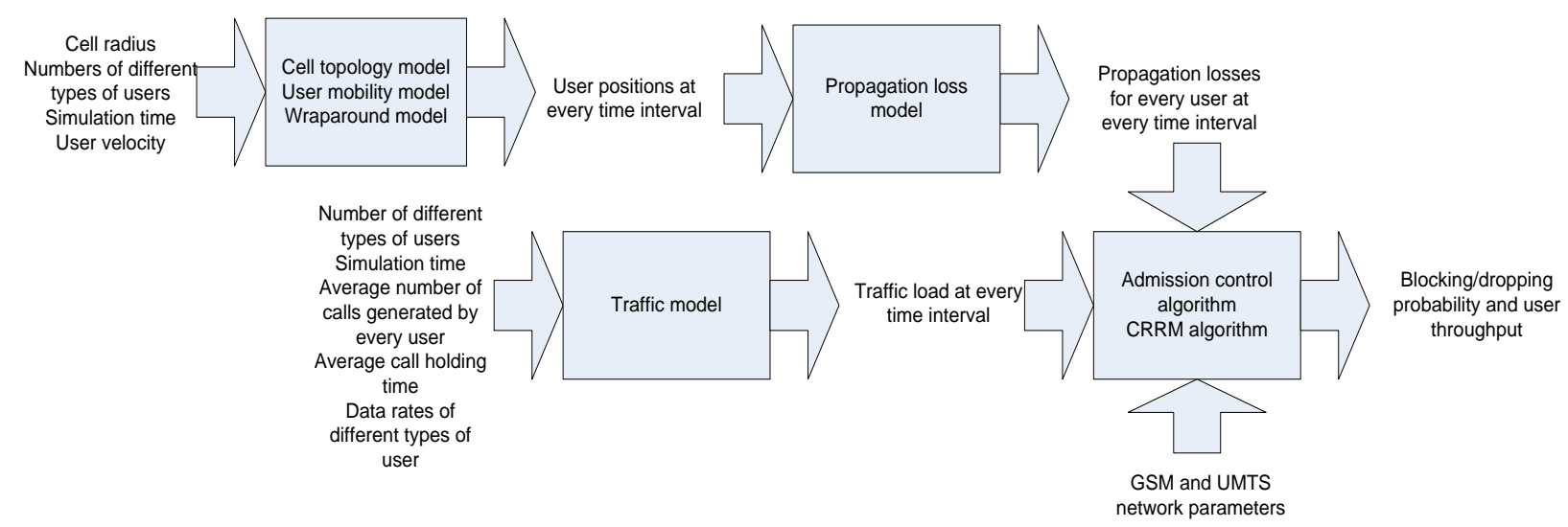

Fig. 2. CRRM simulation model

\section{CRRM SIMULATION MODEL}

In the above sections, different functions modules are described. This section presents the overall CRRM simulation model integrating all the functions modules (as shown in Fig. 2). We take cell radius, numbers of different types of users, simulation time and user velocity as initial inputs and put them into the cell topology model, mobility model and wraparound model functions. The outputs are user positions at every time interval, which are the inputs of the propagation loss model function. The outputs are the propagation loss for every user at every time interval. On the other hand, we take the number of different types of users, simulation time, average number of calls generated by every user, average call holding time, and data rates of different types of user as the inputs of the user traffic model function. The outputs are the traffic loads at every time interval, which are then combined with the GSM and UMTS network parameters and the propagation loss for every user at every time interval as the inputs of the admission control algorithm and CRRM algorithm. The final outputs are the blocking/dropping probabilities and user throughputs, which are the parameters used to evaluate the performance of CRRM algorithm.

\section{CONCLUSIONS AND FUTURE WORKS}

In this paper, the modeling and simulation concepts of our work are presented. The introduced concepts can be used to build a CRRM simulator which takes into account cell topology, user mobility, propagation loss, and admission control. Using the simulator, the performance of different CRRM algorithms can be evaluated. However, in this simulation model, the admission control part only considers a combined GSM/UMTS network case. In our future works, a more generic model including more different kinds of networks will be studied.

\section{ACKNOWLEDGMENT}

This work is partly sponsored by the Commonwealth Scientific and Industrial Research Organisation (CSIRO) Information and Communication Technologies (ICT) centre.
[1] A. Tolli, P. Hakalin, and H. Holma, "Performance Evaluation of Common Radio Resource Management (CRRM)," in IEEE International Conference on Communications, New York, USA, 2002, pp. 34293433.

[2] L. Wu and K. Sandrasegaran, "A Survey on Common Radio Resource Management," in The Second Australia Conference on Wireless Broadband and Ultra Wideband Communications (Auswireless07), Sydney, Australia, 2007, p. 66.

[3] J. Laiho, A. Wacker, and T. Novosad, Radio Network Planning and Optimisation for UMTS: John Wiley \& Sons, 2001.

[4] S. Saunders, Antennas and Propagation forWireless Communication Systems: John Wiley \& Sons, 1999.

[5] 3GPP TR v5.1.0, "RF System Scenarios, Release 5," 2002-06.

[6] H. Holma and A. Toskala, WCDMA for UMTS: radio access for third generation mobile communications, Third edition ed.: John Wiley \& Sons, 2004.

[7] K. Sipila, Z. Honkasalo, J. Laiho-Steffens, and A. Wacker, "Estimation of Capacity and Required Transmission Power of WCDMA Downlink Based on a Downlink Pole Equation," in IEEE Proceeding of 51st. Vehicular Technology Conference Tokyo, Japan, 2000.

[8] J. Schiller, Mobile Communications, 2nd Edition ed. Boston: Addison-Wesley, 2003. 